Resubmitted to: Journal of Experimental Psychology: Human Perception and Performance

\title{
ACQUISITION OF AUTOMATIC IMITATION IS SENSITIVE TO SENSORIMOTOR CONTINGENCY
}

Key words: Automatic imitation, associative sequence learning, mirror neuron system, contingency, sensorimotor learning 


\begin{abstract}
The associative sequence learning model proposes that the development of the mirror system depends on the same mechanisms of associative learning that mediate Pavlovian and instrumental conditioning. To test this model, two experiments used the reduction of automatic imitation through incompatible sensorimotor training to assess whether mirror system plasticity is sensitive to contingency (i.e. the extent to which activation of one representation predicts activation of another). In Experiment 1, residual automatic imitation was measured following incompatible training in which the action stimulus was a perfect predictor of the response (contingent) or not at all predictive of the response (non-contingent). A contingency effect was observed: there was less automatic imitation, indicative of more learning, in the contingent group. Experiment 2 replicated this contingency effect and showed that, as predicted by associative learning theory, it can be abolished by signaling trials in which the response occurs in the absence of an action stimulus. These findings support the view that mirror system development depends on associative learning, and indicate that this learning is not purely Hebbian. If this is correct, associative learning theory could be used to explain, predict and to intervene in mirror system development.
\end{abstract}




\section{INTRODUCTION}

There is now a considerable body of theory and evidence suggesting that learning is critical in the development of the capacity to imitate and of the 'mirror neuron system' (reviewed in: Catmur, Walsh \& Heyes, 2009; Heyes, in press; Ray \& Heyes, under review). However, it is not yet clear what kind of learning is playing this critical role. The purpose of the present study was to find out whether the development of imitation and the mirror neuron system involves domain-general and phylogenetically ancient mechanisms of associative learning; the same mechanisms that produce conditioning phenomena in the laboratory. These mechanisms are known to depend, not only on contiguity, but also on contingency; to become associated, two events must occur together in time and in a predictive relationship (Rescorla \& Wagner, 1972). Therefore, we examined the role of contingency in the learning that modulates 'automatic imitation'.

Automatic imitation is a robust effect found in stimulus-response compatibility paradigms where the stimulus and response sets include the same body movements. For example, Brass, Bekkering, and Prinz (2001) reported that participants made finger lifting responses faster to finger lifting stimuli (compatible trials) than to finger tapping stimuli (incompatible trials). In addition to finger movements, automatic imitation effects have been reported in several other effector systems, including whole hand gestures (e.g. Heyes, Bird, Johnson \& Haggard, 2005), mouth movements (Leighton \& Heyes, in press), and facial expressions (e.g. Dimberg, Thunberg \& Grunedal, 2002). Such effects have been demonstrated for both transitive (object-directed) actions (e.g. Brass et al., 2001) and intransitive actions (e.g. Press, Bird, Walsh \& Heyes, 2008), and can be dissociated from left-right spatial compatibility effects (Bertenthal, Longo \& Kosobud, 2006; Catmur \& Heyes, under review).

The pervasiveness of the human tendency to imitate is further suggested by 'mirror' motor evoked potentials (MEPs) elicited during action observation (e.g. Fadiga, Fogassi, Pavesi \& Rizzolatti, 1995; Strafella \& Paus, 2000). When transcranial magnetic stimulation (TMS) is applied to the primary motor cortex during passive action observation, the amplitude of the MEPs recorded from the muscles required 
to execute that action is greater than the amplitude of the MEPs recorded from task-irrelevant muscles. For example, Strafella and Paus (2000) found that observing handwriting and arm movement stimuli selectively facilitated the amplitude of MEPs recorded from the first dorsal interosseus (responsible for index finger movements) and bicep muscles, respectively. It appears, therefore, that even when actions are not overtly imitated, the passive observation of actions may activate associated motor representations.

Automatic imitation and TMS-induced motor activation during action observation are commonly thought to be mediated by the human mirror neuron system (Catmur, Walsh \& Heyes, 2009). Mirror neurons, which have been identified in the monkey premotor (area F5) and parietal (area PF) cortex, discharge both when the monkey executes an action and when it passively observes an experimenter performing a similar action (e.g. di Pellegrino, Fadiga, Fogassi, Gallese \& Rizzolatti, 1992; Gallese, Fadiga, Fogassi, \& Rizzolatti, 1996; Rizzolatti, Fadiga, Gallese, \& Fogassi, 1996). Brain imaging has revealed similar mirror activity in human premotor and inferior parietal regions; areas considered to be homologous with the macaque mirror areas (e.g. Grèzes, Armony, Rowe \& Passingham, 2003; Iacoboni et al., 1999; Vogt et al., 2007). It has been widely speculated that these areas form part of a circuit, the mirror neuron system, that mediates imitation, action understanding, and other aspects of social cognition (e.g. Gallese, 2001; Gallese \& Goldman, 1998; Rizzolatti \& Craighero, 2004).

Studies of expertise suggest that learning of some kind is important in the development of the mirror neuron system. For example, Haslinger et al. (2005) presented expert pianists and non-pianist controls with sequences of matched finger movements either similar or dissimilar to piano playing. They found that observation of the piano-related stimuli elicited a stronger fMRI response in classical mirror areas (left inferior frontal gyrus, bilateral inferior parietal areas) in the pianists, but not in the non-pianist controls. Similarly, Calvo-Merino, Glaser, Grezes, Passingham and Haggard (2005) showed that mirror system activation was greater when an observer had acquired related motor expertise. Experts in classical ballet, experts in capoeira, and inexpert controls were presented with sequences of capoeira and ballet actions, matched for kinematic properties. Whereas the inexpert controls showed comparable mirror 
activation for both types of stimuli, the dancers showed a clear effect of expertise. For example, a stronger BOLD response was observed in the premotor, parietal and posterior superior temporal sulcus (STS) regions when the ballet experts observed the ballet sequences, than when they viewed the capoeira stimuli. Calvo-Merino, Grezes, Glaser, Passingham and Haggard (2006) subsequently reported that male and female ballet dancers show greater mirror activation when viewing movement sequences unique to their gender, despite equivalent visual exposure to the movements of both sexes. This study is important because it suggests that the effects of expertise on the mirror neuron system do not depend on visual experience alone; that motor experience and/or sensorimotor experience play a critical role.

Training studies have provided evidence that the development of imitation and the mirror neuron system depends specifically on sensorimotor experience. Unlike studies of expertise, which exploit naturally occurring variations in the amount of experience that participants have received in a particular action domain, training studies provide participants with additional experience in the experimental context. Studies of this kind have shown that automatic imitation effects can be enhanced by training in which participants simultaneously observe and execute the same action (Press, Gillmeister \& Heyes, 2007) and abolished by 'incompatible' or 'counter-mirror' training, in which they systematically observe one action while performing another. For example, Heyes et al. (2005) gave participants in one group incompatible training where they were required to open their hand in response to closing-hand stimuli, and vice versa; whereas participants in a second group received compatible training where they simply had to imitate opening- and closing-hand stimuli. When subsequently tested in a stimulus-response compatibility paradigm, the compatible training group showed the typical automatic imitation effect, responding faster to the compatible than to the incompatible stimuli. However, the incompatible training group showed no effect of stimulus compatibility, thus failing to demonstrate automatic imitation.

Similarly, Catmur, Walsh and Heyes (2007) reported that a period of incompatible sensorimotor training reversed muscle-specific responses during observation of little and index finger movements. Prior to training, the typical 'mirror-pattern' was observed: MEPs recorded from the index finger muscle were 
greater during observation of index finger abduction than during observation of little finger abduction, and vice versa for MEPs recorded from the little finger muscle. However, following incompatible training, a counter-mirror pattern was observed such that greater MEPs were recorded from the index finger muscle having observed a little finger movement and vice versa.

Catmur et al. (2008) also reported that a period of incompatible training produced a counter-mirror pattern of fMRI activation. Participants in a compatible training group were required to make foot-lifting responses to a raised-foot stimulus, and hand-lifting responses to a raised-hand stimulus. Conversely, participants given incompatible training had the opposite pattern of stimulus-response mappings. Comparison of the groups following training revealed that the relative action observation properties of the mirror system were reversed. Those areas which showed greater fMRI responses to observed hand actions in the compatible training group, responded more strongly to observed foot movements in the incompatibly trained group.

The evidence described above suggests that there is little or nothing that is intrinsically 'mirror' about the mirror system. Rather, so-called "mirror" neurons may acquire various response profiles depending on the nature of the correlated sensorimotor experience received in the course of development. However, although considerable evidence has accumulated implicating sensorimotor learning in mirror system development, at present relatively little is known about the mechanisms mediating this learning.

The associative sequence learning model (ASL) (Heyes, 2001; Heyes \& Ray, 2000) proposes that the development of the mirror system is mediated by phylogenetically ancient mechanisms of sensorimotor learning - the very same mechanisms of associative learning that produce Pavlovian and instrumental conditioning in human and nonhuman animals. If this is correct, associative learning theory - the body of knowledge accumulated through the use of conditioning procedures - could be used to explain, predict and potentially to intervene in the development of imitation and the mirror system. Studies of instrumental learning have identified two main determinants of the acquisition of associations between 
stimuli (Ss) and responses (Rs): the temporal contiguity between stimulus and response and their contingency. Imposing a delay between a stimulus and a response decreases learning in proportion to the delay in both animals (Dickinson, Watt, \& Griffiths, 1992) and humans (Shanks \& Dickinson, 1991). However, if learning occurs whenever a stimulus and response are paired in time, the animal would be prone to acquiring superstitious associations. This point can be illustrated by considering a case in which the contribution of temporal contiguity to learning can be kept constant by fixing the probability that the stimulus is paired with the response. However, if the response is just as likely to occur in the absence of the stimulus as in its presence, the stimulus does not predict an increase in the likelihood of the response above its base rate and, therefore, is uninformative about the occurrence of the response irrespective of the number of times that they are paired. In order to ensure that the strength of an association reflects the predictive relationship between stimulus and response, the learning mechanism must be sensitive not only to the probability of the response given the stimulus $[\mathrm{P}(\mathrm{R} / \mathrm{S})]$, but also to the probability of the response in the absence of the stimulus $[\mathrm{P}(\mathrm{R} / \sim \mathrm{S})]$ or, in other words, to the contingency between the stimulus and response.

Both animal (e.g. Dickinson \& Charnock, 1985) and human instrumental learning (e.g. Shanks \& Dickinson, 1991; Elsner \& Hommel, 2004) is sensitive to the contingency between stimulus and response. In these studies the probability of stimulus-response pairings was fixed to ensure a constant number of contiguous pairings, while the contingency was degraded by increasing the probability of unpaired presentations. Whereas learning was observed when the probability of unpaired presentations was low, and therefore the contingency high, little or no learning occurred in a non-contingent condition in which the probabilities of paired and unpaired presentations were the same. In these studies of instrumental learning, the stimulus acted as an outcome of the response. However if ASL is correct in its claim that common associative mechanisms mediates both forms of learning, then the same sensitivity to the contingency between stimulus and response should be observed in the counter-mirror training paradigm, where the stimulus is antecedent to the response. To examine this prediction, Experiment 1 sought to determine whether the reduction of automatic imitation is sensitive to variations in contingency 
by measuring residual imitation effects following either contingent incompatible training (where the stimulus was a perfect predictor of response) or non-contingent incompatible training (where the stimulus did not predict an increase in the likelihood of the response). Having observed an effect of contingency in the first experiment, Experiment 2 analyzed the mechanism by which contingency acts upon sensorimotor learning through the addition of a third, 'signaled' training variant.

\section{EXPERIMENT 1}

In order to determine whether the sensorimotor learning exhibited by the mirror system is sensitive to variations in sensorimotor contingency, residual automatic imitation effects were measured following two sessions of either contingent or non-contingent counter-mirror training. During training, participants were given a choice reaction-time (RT) task in which hand-open responses were made to closed-hand stimuli and hand-close responses made to open-hand stimuli. This was achieved through the use of a compound stimulus comprising a task irrelevant hand stimulus (depicting either an open- or closed-hand) and a numerical imperative stimulus (either 1 or 2). Participants thus responded to the number presented, but were exposed to incompatible visual stimuli. The number of contiguous sensorimotor pairings was held constant across the training groups, in that both groups made equal numbers of open and close responses in the presence of the incompatible (and irrelevant) hand stimuli (hereafter 'paired trials'). However, the sensorimotor contingencies between the incompatible hand stimuli and the responses were degraded for the non-contingent group through the addition of trials in which responses were made in the absence of an incompatible hand stimulus (hereafter 'unpaired' trials').

Residual automatic imitation was subsequently assessed using a simple RT task. Starting from a neutral hand gesture, participants were required to make a pre-specified response (e.g. open) to the onset of compatible (open) and incompatible (closed) stimuli. The magnitude of the residual imitation effect was calculated by subtracting the mean RT on compatible trials (open responses to open stimuli and close responses to closed stimuli) from the mean RT on incompatible trials (open responses to closed stimuli and closed responses to open stimuli). 
If the development of imitation and the mirror system is mediated by associative learning, the effects of sensorimotor training should be sensitive to contingency. Thus, the ASL model predicts that the contingent training condition will produce greater incompatible learning, and hence less automatic imitation, than non-contingent training. Conversely, if equivalent sensorimotor learning is observed in the contingent and non-contingent groups, this would indicate that the learning involved in mirror system development is sensitive to contiguity only, as the number of contiguous stimulus-response pairings is held constant across training conditions. This outcome would therefore imply that mirror system development is not mediated by the kind of associative learning observed in conditioning experiments.

\section{Method}

\section{Participants}

Twenty-four healthy adults ( 5 male) with a mean age of 23.0 years served as participants in the experiment in return for a small honorarium. All were right handed, had normal or corrected-to-normal vision, and were naive to the purpose of the experiment. Participants were assigned randomly to either the contingent or non-contingent training groups in equal numbers. Two were replacements for participants who responded slowly during the test phase (RT $>400 \mathrm{~ms})$. The study was approved by the University College London ethics committee and performed in accordance with the ethical standards set out in the 1964 Declaration of Helsinki.

\section{Apparatus and stimuli}

The stimuli used in the training phase consisted of 12 digital images; 8 for use on paired trials, and 4 for use on response-only trials (see Figure 1). Each depicted a model's right hand in naturalistic tones against a black background. Of the 8 images used on the paired trials, 4 showed an open hand, and 4 a closed hand. The open stimulus showed fingers and thumb splayed. The male stimulus subtended approximately $17^{\circ}$ of visual angle horizontally and $20^{\circ}$ vertically and the female stimulus subtended approximately $16^{\circ}$ of visual angle both horizontally and vertically. The closed gesture was a fist. The 
male stimulus subtended approximately $11^{\circ}$ horizontally and $14^{\circ}$ vertically, and the female stimulus subtended approximately $8^{\circ}$ horizontally and $12^{\circ}$ vertically. Four images depicted a male hand and 4 a female hand. The 4 stimuli used on the response-only trials each presented a neutral hand gesture, with fingers together and pointing upwards in parallel with the thumb. Two of the images depicted a male hand and 2 a female hand. The imperative stimulus was a white number, either a 1 or a 2, presented centrally in Arial font, subtending approximately $1.75^{\circ}$ vertically.

Four similar digital images were used during the test phase. The same male and female hands were shown, depicting either an open or closed gesture. However, in contrast to the images used during training, the test stimuli did not have a number superimposed.

Two further images were employed during training and test phases as warning stimuli. These images depicted the same neutral hands, either male or female, as the stimuli presented on the response-only trials, but without a superimposed numerical stimulus. Both training and test trials were presented in colour on an IBM compatible laptop computer with a 38-cm screen (resolution 1024 x 678).

\section{Figure 1 about here}

\section{Data recording and analysis}

Both the training and test procedures took the form of RT tasks where electromyography (EMG) was used to establish response onset. Recordings were taken from the first dorsal interosseus (FDI) muscle using disposable $\mathrm{Ag} / \mathrm{AgCl}$ surface electrodes. The EMG signal was amplified, mains-hum filtered at 50 $\mathrm{Hz}$ and digitized at $2.5 \mathrm{kHz}$. They were rectified and smoothed using a dual-pass Butterworth filter, with cut-off frequencies of $20 \mathrm{~Hz}$ and $1000 \mathrm{~Hz}$. The EMG signal was registered for $100 \mathrm{~ms}$ before the onset of the imperative stimulus to define the baseline. A window of $20 \mathrm{~ms}$ was then shifted incrementally over the raw data in 1-ms steps. Response onset was defined as the start of the earliest 20-ms window, following presentation of the imperative stimulus, in which the standard deviation for that window, and 
for the following 20-ms epoch, was greater than 2.75 times the standard deviation of the baseline. That this criterion reasonably defined gesture onset was verified by sight for every training and test trial.

\section{Procedure and design}

The experiment was conducted over 3 sessions each completed approximately 24-hours apart in a dimly lit room. In all 3 sessions, participants sat at a viewing distance of approximately $60 \mathrm{~cm}$ with their shoulders and right forearm parallel to the stimulus display. Their elbow and forearm were supported by an armrest, with both the hand and forearm occluded from view. The participant's hand and wrist were positioned such that their fingers moved upwards during open responses and downwards during close responses. Stimulus postures were presented in the lateral plane (left-right), thus ensuring response movements were orthogonal to stimulus postures throughout. This feature of the design allows automatic imitation to be isolated from left-right spatial compatibility.

The training procedure took the form of a choice RT task (see Figure 2). The contingent and noncontingent training variants each consisted of 6 blocks of incompatible training trials spread equally over 2 sessions, completed approximately 24-hours apart. On each trial, participants were required to make one of the two speeded response movements (open or close) as soon as the numerical imperative stimulus appeared, and then to return to the starting position ready for the next trial. Whether participants were required to make open or close responses to either 1 or 2 was counterbalanced across training conditions. The order of stimulus presentation during the training trials was randomised. The beginning of each trial was indicated by the appearance of the warning stimulus, which was then replaced by one of the training stimuli, presented for 480ms. Stimulus onset asynchrony (SOA) was varied randomly between 800 and 1500 in $50 \mathrm{~ms}$ increments. The hand depicted in the warning stimulus was identical to that in the subsequent training stimuli, thus giving rise to apparent motion. On paired trials, the compound training stimulus consisted of an imperative stimulus (a ' 1 ' or a '2') superimposed on a taskirrelevant action image (an open or closed hand). On response-only trials, the imperative stimulus was 
superimposed on the image of the neutral hand stimulus. After the training stimulus, the screen went blank for 3000ms, until the warning stimulus for the subsequent trial was presented.

For the contingent group, each training block comprised 72 paired trials (Figure 2a). On 36 trials participants were required to make close responses having seen an open stimulus, and on 36 trials they made open responses having seen a closed stimulus. Thus, for the contingent group, there was a perfect contingency between the irrelevant stimuli and the counter-mirror responses $[P(R / S)=1 ; P(R / \sim S)=0]$. Open and closed stimulus trials were presented in random order. Prior to each of the two sessions of training, participants in the contingent group completed 4 practice trials.

For the non-contingent group, each training block comprised 144 trials (72 paired, 72 unpaired). On 36 of the paired trials participants were required to make close responses having seen an open stimulus, and on 36 trials they made open responses having seen a closed stimulus. Consequently, the number of temporally contiguous sensorimotor pairings was held constant across both training conditions. However, the non-contingent training contingency was degraded through the addition of 72 unpaired trials in which responses were elicited from participants in the absence of an incompatible irrelevant stimulus (see Figure 2b). On 36 of the unpaired trials participants had to make open responses to the imperative stimulus, and on 36 of the unpaired trials they had to make close responses. Consequently, during non-contingent training, there was no contingency between the irrelevant stimuli and the responses because the responses were equally likely to occur in the presence and in the absence of the incompatible stimuli $[\mathrm{P}(\mathrm{R} / \mathrm{S})=\mathrm{P}(\mathrm{R} / \sim \mathrm{S})=1]$. Open and closed stimulus trials were again presented in random order. Whether trials were paired or unpaired was also randomised. Because the non-contingent blocks were longer, participants were given an opportunity to take a short break after the first 72 trials. Prior to each non-contingent training session 8 practice trials were completed.

\section{Figure 2 about here}


Approximately 24-hours after completion of the second training session, both groups were given identical test procedures to assess the impact of the two training variants on participants' compatibility effects. The test was a simple RT task, in which EMG recording was again used to establish response onset. The test comprised two blocks of 120 trials during which participants made speeded pre-specified movements (open responses in one block, close in the other) in response to the onset of open and closed stimuli (see Figure 3). On each stimulus trial, participants started in the neutral hand position, were required to make their response as soon as a gesture stimulus appeared and then return to the neutral position ready for the next trial. The gesture stimulus was present for $480 \mathrm{~ms}$ until replaced by a blank display for $3000 \mathrm{~ms}$, prior to the warning stimulus for the next trial. SOA was varied randomly between 800 and 1500 in 50ms increments. Twenty-four catch trials were included in each test block, in which the warning stimulus was displayed throughout the trial and participants had been instructed to make no response whatsoever. On catch trials, the warning stimulus was presented for $1980 \mathrm{~ms}$ before the 3000 ms inter-trial interval. The order in which participants completed the open and close blocks was counterbalanced across conditions.

\section{Results and Discussion:}

Training and test trials in which participants made incorrect responses, no response at all, or where the time of movement onset was equivocal were excluded from all further analyses ( $2.5 \%$ of training trials; $1.6 \%$ of test trials). Any remaining data points beyond 2.5 standard deviations of a participant's mean response latency for a given block were also excluded (2.1\% of training trials; $1.8 \%$ of test trials). Participants' EMG signals on catch trials were examined to ensure that they obeyed task instructions, but were excluded from all further analyses. During the test sessions, participants initiated movements on only $5.8 \%$ of catch trials, implying that they were not making anticipatory responses on the stimulus trials.

\section{Training}


Figure 4 shows mean RTs for the contingent and non-contingent groups. A mixed model ANOVA with training block as a within subjects factor and training group as a between subjects factor revealed a significant linear decline across the 6 training blocks $\left[F(1,22)=8.6, p<.01, \eta^{2}=.281\right]$ suggestive of learning. No group $\mathrm{x}$ block interaction was observed $\left[F(1,22)=0.4, p>.51, \eta^{2}=.019\right]$, indicating that the improvement in training task performance was comparable across groups. Equally, no main effect of group was present $\left[F(1,22)=0.04, p>.84, \eta^{2}=.002\right]$ implying that overall reaction times during the training task were also equivalent. Simple effects analysis revealed that the difference between the groups at day-1 block-1 was not significant $[t(22)=0.3, p>.75]$, indicating no difference in baseline performance.

\section{Figure 4 about here}

\section{Test}

Figure 5 shows (a) the mean RTs on compatible and incompatible trials and (b) the mean automatic imitation effects for the two training groups. These data were analysed using a mixed-model ANOVA with trial type (compatible or incompatible) as a within-subjects factor and training group (contingent or non-contingent) as the between-subjects factor. The analysis revealed a significant main effect of trial type $\left[F(1,22)=159.9, p<.001, \eta^{2}=.879\right]$, indicating that compatible responses $($ mean $=263.4 \mathrm{~ms})$ were executed faster than incompatible responses $($ mean $=284.7 \mathrm{~ms})$. Crucially, the interaction between trial type and training condition was also significant $\left[F(1,22)=5.8, p<.05, \eta^{2}=.209\right]$. This indicates that the effect of trial compatibility (automatic imitation) was greater in the non-contingent training group (mean $=25.3 \mathrm{~ms})$ than in the contingent training group (mean $=17.2 \mathrm{~ms})$. No main effect of group was observed $\left[F(1,22)=1.5, p>.23, \eta^{2}=.064\right]$, indicating that the small disparity in mean RTs between the contingent group $($ mean $=261.8 \mathrm{~ms})$ and non-contingent group $($ mean $=286.3 \mathrm{~ms})$ was not significant.

\section{Figure 5 about here}


In order to determine how residual compatibility effects varied across the distribution of RTs, each participant's distribution of compatible and incompatible RTs was split into four quartiles. Four further compatibility effects were then calculated by subtracting the mean RT for a given quartile of the compatible distribution from the mean RT of the corresponding quartile of the incompatible distribution (Figure 6). The magnitudes of these compatibility effects were then analysed using a mixed-model ANOVA with quartile as a within subjects factor and group as a between subjects factor. The analysis not only revealed a significant main effect of group $\left[F(1,22)=4.5 ; p<.05, \eta^{2}=.170\right]$ but also a significant linear trend across quartile $\left[F(1,22)=8.3 ; p<.01, \eta^{2}=.274\right]$ suggesting that slower responding was associated with a stronger tendency to imitate. Of particular interest, no quartile x group interaction was observed $\left[F(3,66)=.01 ; p>.90, \eta^{2}=.004\right]$ indicating that the differential effect of contingent and non-contingent training was observed across the entire distribution of reaction times.

\section{Figure 6 about here}

Given that all participants received incompatible training - training which, if effective, would tend to reduce automatic imitation - the smaller automatic imitation effect found in the contingent group implies that more learning occurred in this group than in the non-contingent group. An associative account suggests that, as a result of training, the contingent group acquired stronger incompatible associations (between the open stimulus and close response, and between the close stimulus and open response) than did the non-contingent group. Assuming that, before training, the two groups had equally strong compatible associations (between the open stimulus and open response, and between the close stimulus and close response), stronger incompatible associations would overcome more completely the effects on behaviour of pre-existing, compatible associations, and therefore result in more complete abolition of the automatic imitation effect.

The difference between the contingent and non-contingent groups was robust across the RT distribution. This is of interest because it makes 'strategic' or 'top-down' explanations for the group difference 
unlikely. For example, although all participants were instructed to respond to the digit during training, it is possible in principle that participants in the contingent group formulated and used a 'do opposite' response rule. This strategy - responding to open stimuli with close responses and vice versa - would have enabled correct responding during training in the contingent group but not in the non-contingent group, and interference from such a response rule could have reduced the magnitude of the automatic imitation effect on test. However, given that strategic processing is relatively slow (Kornblum, Hasbroucq \& Osman, 1990), if it was a strategic process, rather than learning of incompatible associations, that reduced the automatic imitation effect in the contingent group, one would have expected the group difference to have increased with RT. Thus, the fact that the effect of contingency did not vary across the RT distribution supports the view that it was due to superior learning of incompatible associations during training, and their automatic activation in the test phase.

In the absence of a no-training control group, we cannot say with certainty whether the non-contingent group showed any counter-mirror sensorimotor learning. The automatic imitation effect observed in the non-contingent group $($ mean $=25.3 \mathrm{~ms}$ ) was smaller than that reported by Heyes et al. (2005) following 6 blocks of 72 compatible training trials (mean $=34.0 \mathrm{~ms}$ ). It should be noted, however, that unlike the training procedure employed here, the 6 blocks administered by Heyes et al. were completed in a single training session, 24 hours before the test procedure. Nevertheless, this comparison suggests that little, if any, learning took place in the non-contingent group, whereas contingent training resulted in a substantial reduction in the compatibility effect $($ mean $=17.2 \mathrm{~ms})$.

Although the results of this experiment suggest that the sensorimotor learning exhibited by the mirror system is sensitive to the stimulus-response contingency, our procedure confounds the stimulus-response contingency with the number of times the participants performed the responses. Members of the noncontingent group performed each response twice as often as those in the contingent group. If repetition habituates the activation of a representation of a response by its execution, the impaired learning observed in the non-contingent group could have been due to enhanced habituation rather than the 
degradation of the stimulus-response contingency. According this habituation account, the activation of a representation of hand-opening or -closing response on a paired trial would have been reduced in the non-contingent group due to the habituation produced by the execution of these responses on the unpaired trials. Thus the sensorimotor learning on the paired trials may have been reduced by the habituation produced on the unpaired trials. The second experiment examined the role of an habituation process in the contingency effect.

\section{EXPERIMENT 2}

The best evidence that contingency sensitivity in both animal (e.g., Dickinson \& Charnock, 1985) and human instrumental learning (e.g. Shanks, 1989) is not due to habituation comes from studies investigating the effect of introducing an extraneous signal on the unpaired trials. The rational for this signaled condition can be illustrated by reference to the counter-mirror paradigm used in Experiment 1. Although the various associative theories explain contingency sensitivity through different mechanisms (see General Discussion), in one way or another they all appeal to the idea that when two or more stimuli are present on a trial, they compete for the acquisition of associative strength and thereby for predictive learning. Moreover, the greater the associative strength of a stimulus the more effectively it competes with other stimuli present on the trial. In the case of our counter-mirror paradigm, the target stimuli, the opening and closing of the hand, should have suffered competition from the warning stimulus, the static hand stimulus. Because the static hand warning stimulus was present on unpaired trials the associative strength of this warning stimulus for each of the responses should have been enhanced in the noncontingent group. This, in turn, should have augmented the ability of the warning stimulus to compete with the target opening- and closing-hand stimuli on the paired trials, and thereby reduced control over the response by these stimuli in the non-contingent condition.

This competition can be ameliorated, however, by signaling the responses on the unpaired trials with a warning stimulus that differs from that employed on the paired trials. In this signaling condition, the unpaired trials do not enhance learning about the warning signal employed on the paired trials. 
Therefore, the competition on these trials is effectively the same as that experienced in the contingent condition, without any unpaired trials. By contrast, the habituation account predicts that the impact of unpaired trials should be equivalent in the non-contingent and signaled conditions because the two responses are performed exactly the same number of times and at the same frequency in the two conditions.

Experiment 2 examined the contrasting predictions of the habituation and associative accounts not only by replicating the contingent and non-contingent conditions from Experiment 1, but also by adding a signaling condition in which the warning stimulus on unpaired trials differed from that on paired trials. According to the simple habituation account, the inclusion of unpaired trials should have degraded counter-mirror learning as effectively in the signaled condition as under the non-contingent schedule. In contrast, associative competition predicts that the presence of a different warning stimulus on the paired and unpaired trials should have enabled the signaling condition to sustain learning equivalent to that observed in the contingent condition.

\section{Method}

\section{Participants}

A further 36 healthy adults (13 male) with a mean age of 25.4 years served as participants in the experiment in return for a small honorarium. All were right handed, had normal or corrected-to-normal vision, and were naive with respect to the purpose of the experiment. Participants were assigned randomly to either the contingent, non-contingent or signaled training groups in equal numbers. Five were replacements for participants who responded slowly during the test phase (RT > 400ms).

\section{Apparatus and stimuli}

The training stimuli used in the contingent and non-contingent groups were the same as those used in Experiment 1 . However, the addition of the signaled training condition required a further 4 stimuli to act as contextual signals on the response-only trials. Each of these images depicted a neutral hand (either 
male or female) coloured blue against a red background (see Figure 7). Again, a numerical imperative stimulus (either a 1 or a 2) was superimposed centrally. Each response-only stimulus was followed by an audible $880-\mathrm{Hz}$ tone, presented for $250 \mathrm{~ms}$ immediately following the imperative stimulus. A further 2 warning stimuli were also employed depicting the male and female hands in blue against a red background. The stimuli used during the test phase were identical to those described in Experiment 1.

\section{Figure 7 about here}

\section{Procedure and design}

The training procedure used for the contingent and non-contingent groups was the same as in Experiment 1. The procedure employed in the signaled condition was virtually identical to that for the non-contingent group, the only difference being that the contextual signals were presented on the response-only trials (see Figure 8). As in Experiment 1, whether participants were required to make open or close responses to either 1 or 2 was counterbalanced across training groups, as was the order in which participants completed the two test blocks. The test procedure was identical to that employed in Experiment 1.

\section{Figure 8 about here}

\section{Results and discussion}

Training and test trials where participants made incorrect responses, made no response or where the precise movement onset was equivocal were again excluded from all further analyses $(3.3 \%$ of training trials; $2.6 \%$ of test trials). Any remaining data points beyond 2.5 standard deviations of a participant's mean response latency for a given block were also excluded (2.0\% of training trials; $2.1 \%$ of test trials). During the test sessions, participants initiated movements on only $7.5 \%$ of catch trials.

\section{Training}


Figure 9 shows mean training RTs for the contingent, non-contingent and signaled groups. A mixed model ANOVA with training block as a within subjects factor and training group as a between subjects factor revealed a significant linear decline across the 6 training blocks $\left[F(1,22)=4.3, p<.05, \eta^{2}=.116\right]$

suggestive of learning. No group x block interaction was observed $\left[F(10,165)=0.6, p>.83, \eta^{2}=.034\right]$ indicating that this improvement in performance was comparable across groups. Similarly, no main effect of group was present $\left[F(2,33)=2.0, p>.14, \eta^{2}=.109\right]$ implying that overall reaction times during the training task were equivalent. Simple effects analysis revealed that the difference between the groups at day-1 block-1 was not significant $[F(2,33)=1.0, p>.36]$, indicating no pre-existing difference in baseline performance.

\section{Figure 9 about here}

\section{Test}

Figure 10 shows (a) the mean RTs on compatible and incompatible trials and (b) the mean automatic imitation effects for the three training groups. These data were analysed using a mixed-model ANOVA with trial type (compatible or incompatible) as a within subjects factor and training variant (contingent, non-contingent and signaled) as the between subjects factor. A significant main effect of trial type was observed $\left[F(1,33)=112.7, p<.001, \eta^{2}=.773\right]$ indicating that compatible responses $($ mean $=246.2 \mathrm{~ms})$ were executed faster than incompatible responses (mean $=270.6 \mathrm{~ms}$ ). The analyses revealed no significant main effect of group, indicating that the small differences in mean RTs between the contingent $($ mean $=262.8 \mathrm{~ms})$, non-contingent $($ mean $=267.4 \mathrm{~ms})$, and signaled groups $($ mean $=245.0 \mathrm{~ms})$ were not significant. As in Experiment 1, the crucial interaction between trial type and training condition was significant $\left[F(2,33)=4.2, p<.025, \eta^{2}=.202\right]$, indicating that the effect of compatibility (automatic imitation) differed across groups. In order to interpret this $2 \times 3$ interaction, three further $2 \times 2$ interactions were calculated. A significant interaction was observed between trial type and training (contingent vs. non-contingent) $[F(1,33)=5.2, p<.05]$ indicating greater automatic imitation in the non-contingent 
condition $($ mean $=33.6 \mathrm{~ms})$ than in the contingent condition $($ mean $=20.8 \mathrm{~ms})$. A significant interaction was also observed between trial type and training (signaled vs. non-contingent) $[F(1,33)=7.2, p<.025]$ indicating greater automatic imitation in the non-contingent condition than in the signaled condition $($ mean $=18.6 \mathrm{~ms})$. However, no significant interaction was observed between trial type and training (contingent vs. signaled) indicating that automatic imitation effects were comparable for the contingent and signaled conditions.

\section{Figure 10 about here}

As in Experiment 1, the participants' distribution of compatible and incompatible responses were additionally split into four quartiles in order to assess how the residual imitation effects varied across the distribution of RTs (see Figure 11). Having calculated 4 new imitation effects for each subject (one for each quartile), the size of the effects were analysed using a mixed-model ANOVA with quartile as a within subjects factor and group as a between subjects factor. In addition to a significant main effect of group $\left[F(2,33)=4.201 ; p<.025, \eta^{2}=.203\right]$, the analysis revealed a significant linear trend across quartile $\left[F(1,33)=12.056 ; p<.001, \eta^{2}=.268\right]$ suggesting that slower responding was associated with a stronger tendency to imitate. Consistent with Experiment 1, no quartile $\mathrm{x}$ group interaction was observed $[F(6,99)$ $\left.=.974 ; p>.440, \eta^{2}=.056\right]$ indicating that the differential learning effects were again seen across the entire range of reaction times.

\section{Figure 11 about here}

This pattern of results replicates that observed in Experiment 1, in that participants given contingent training showed smaller automatic imitation effects than those given non-contingent training. Moreover, the signaled group showed automatic imitation effects equivalent to the contingent training group, but significantly smaller than the non-contingent group. The fact that the contingent and signaled groups showed smaller automatic imitation effects suggests that the participants in these groups learned more 
during training than the participants in the non-contingent group, thereby acquiring stronger incompatible associations. Thus, when unpaired, response-only trials were signaled, they did not impair learning.

The fact that the contingent and signaled groups showed less residual automatic imitation suggests that participants in these groups learned more during training - acquiring stronger incompatible associations than participants in the non-contingent group. Interestingly, the magnitude of the residual automatic imitation effects observed for the non-contingent group $($ mean $=33.6 \mathrm{~ms})$ was almost identical to that reported by Heyes et al. (2005) following six block of 72 compatible training trials (mean $=34.0 \mathrm{~ms}$ ). This suggests that the participants in the non-contingent group in Experiment 2 may have learned nothing about the irrelevant stimulus during training.

That signaling the response with a different warning stimulus on unpaired trials attenuates their detrimental effect on stimulus-response learning suggests that the basic contingency effect observed in Experiment 1, and replicated in Experiment 2, is not mediated by simple response habituation. Rather the contingency effect arises from the type of stimulus competition observed in other associative learning paradigms. In the standard non-contingent condition, the unpaired trials served to enhance the associative strength of the warning stimulus, which then competed more effectively with the target opening- and closing-hand stimuli for control over the respective responses. The use of a different warning stimulus on unpaired trials in the signaled condition alleviated this competition and restored learning to the level observed in the contingent condition.

\section{GENERAL DISCUSSION}

There is now a substantial body of evidence suggesting that the mirror system may be modified through correlated sensorimotor experience, but little is known about processes involved in this learning. The aim of the present study was to assess the suggestion that the development of the mirror system depends on the same mechanisms of associative learning that mediate Pavlovian and instrumental conditioning 
(Heyes, 2001; Heyes \& Ray, 2000), and in particular to establish whether it is sensitive to variations in contingency.

In Experiment 1, residual automatic imitation effects were measured following either contingent or noncontingent incompatible training. Whereas the number of temporally contiguous stimulus-response pairings was held constant across training groups, the stimulus-response contingency was degraded in the non-contingent group through the addition of response-only unpaired trials, in which participants made motor responses in the absence of an action stimulus. A contingency effect was observed: participants given non-contingent training demonstrated larger automatic imitation effects than those given contingent training. In Experiment 2, this contingency effect was replicated: participants given noncontingent training again showed larger automatic imitation effects than those who received contingent training. However, in addition to the original two training groups, Experiment 2 assessed learning in a third 'signaled' training group, for whom the warning stimulus of the response-only trials was differentiated from that of the stimulus-response trials. Participants in the signaled group demonstrated smaller automatic imitation effects than participants in the non-contingent group, but equivalent to those of the contingent group. The smaller automatic imitation effects in the signaled and contingent groups provide evidence of superior learning. Participants in these groups seemingly acquired during the training phase stronger associative links between the open and close motor responses and the incompatible visual stimuli. These links subsequently facilitated faster responding on the incompatible test trials, thus resulting in smaller automatic imitation effects.

The finding that automatic imitation is modified by relatively brief periods of incompatible training is consistent with several previous findings (e.g. Catmur et al., 2007; Gillmeister, Catmur, Liepelt, Brass, \& Heyes, 2008; Heyes et al., 2005). Given that automatic imitation is thought to be a behavioural product of the mirror system, these findings therefore add to the body of evidence suggesting that the sensorimotor properties of the mirror system are acquired ontogenetically. Whether human and monkey infants are predisposed to acquire mirror links remains an open question. However, if the properties of 
the mirror system were innate, one might expect them to show more resistance to the counter-mirror effects of incompatible sensorimotor training (e.g. Heyes, in press; Pinker, 1997).

The ASL model proposes that the development of the mirror system depends on the same mechanisms of associative learning that mediate conditioning in human and nonhuman animals (Heyes, 2001; Heyes \& Ray, 2000). It has been known for at least 40 years that these mechanisms depend on contingency (Rescorla, 1968). Therefore, the ASL model directly implies that the development of the mirror system depends on contingency. Another model, the 'Hebbian perspective' (Keysers \& Perrett, 2004; Keysers \& Gazzola, 2006), also suggests that mirror neuron development depends on some kind of associative process. However, the name of this model indicates that it is based on the principles advanced by Hebb (1949), and these principles assume that contiguity is sufficient for associative learning. This is evident in Hebb's famous dictum 'Cells that fire together, wire together', and was more formally stated: "any two cells or systems of cells that are repeatedly active at the same time will tend to become 'associated,' so that activity in one facilitates activity in the other" (Hebb 1949, p70). Thus, the 'Hebbian perspective' on mirror neurons implies that contiguity is sufficient for mirror neuron development; that mirror neuron development does not also depend on contingency.

The contingency effects observed in Experiments 1 and 2 do not accord with this view. A model based solely on Hebbian principles predicts equivalent learning across training conditions where the number of contiguous stimulus-response pairings is held constant. However, in both Experiments 1 and 2, the contingent group showed greater sensorimotor learning than the non-contingent group, despite both receiving the same number of temporally contiguous stimulus-response pairings during training. This finding suggests that the learning exhibited by the mirror system is sensitive, not only to sensorimotor contiguity, but also to sensorimotor contingency.

A purely Hebbian account of the contingency effect observed in Experiment 1 can be sustained if it is assumed that extinction occurs in response-only unpaired trials due to an anti-Hebbian process. This 
account predicts that signaling the response-only trials should have no influence on the strength of learning, because, even when such trials are signaled, they involve the execution of responses in the absence of their incompatible visual stimuli. In other words, in spite of signaling, the anti-Hebbian process should continue to act against the learning taking place on the stimulus-response trials. However, this prediction was not supported by the signaling effect observed in Experiment 2: the signaled group showed more learning than the non-contingent group. Therefore, it seems that a sensorimotor learning account based solely on Hebbian principles is unable adequately to explain the contingency effects observed in the present study.

In contrast, the fact that the sensorimotor learning exhibited by the mirror system is sensitive to variations in contingency is consistent with the ASL model (Heyes, 2001; Heyes \& Ray, 2000). The ASL model is similar to the subsequently developed Hebbian perspective in that the latter also regards the concurrent activation of sensory and motor representations as an important determinant of sensorimotor learning. However, the ASL model proposes that the development of the mirror system depends on the standard associative principles developed through research using conditioning procedures. Therefore, ASL predicts that the sensorimotor learning responsible for formation of the mirror system will have characteristic properties in common with the associative learning that mediates conditioning; properties such as a sensitivity to contingency. The ASL framework seems, therefore, to offer a better account than the Hebbian perspective of the training effects observed in our experiments.

When applied to the present sensorimotor paradigm, theories of associative learning assume that when two or more stimuli are paired with a response, these stimuli compete for association with the response, either directly (Rescorla \& Wagner, 1972; Wagner, 1981) or through attentional processes (Mackintosh, 1975; Pearce \& Hall, 1980). Therefore, in our counter-mirror paradigm the warning signal competes with the irrelevant action stimuli for association with the hand-opening and-closing responses on the paired trials. In the non-contingent condition, the competition from the warning stimulus on the paired trials is enhanced by the extra associative strength acquired by the warning stimulus on the unpaired trials, 
thereby reducing the strength of the associations formed between the action stimuli and the responses. This extra source of competition is removed in the signaling conditioning because the warning stimulus on the unpaired trials differs from that on the paired trials.

We have interpreted the training effects observed in Experiments 1 and 2 as evidence of learning within the human mirror system. However, one might reasonably argue that, rather than modifying the mirror system directly, sensorimotor training sets up additional stimulus-response associations which act in parallel to the mirror system. For example, both neurophysiological data from monkeys (Halsband \& Passingham, 1985; Mitz, Godschalk \& Wise, 1991; Petrides, 1986) and human neuroimaging studies (Grafton, Fagg \& Arbib, 1998) suggest that dorsal premotor cortex may support stimulus-response learning; an area not typically regarded as part of the human mirror system. However, there are at least three reasons to doubt this alternative interpretation. First, the stimulus-response associations supported by dorsal premotor areas are typically between abstract visual stimuli (colours, symbols, characters) and related responses. In contrast, associations acquired during counter-mirror training are not between abstract stimuli and responses, but between a biological action stimulus and a response, and are thus likely to receive their visual input from distinct parts of the visual system (e.g. Oram \& Perrett, 1994). Second, Catmur et al. (2008) have demonstrated that counter-mirror training is associated with fMRI signal changes in classical mirror areas. Peak voxels in ventral premotor and inferior parietal regions, which showed greater responses to observation of hand actions following compatible training, responded more strongly to observation of foot actions following incompatible, counter-mirror training. Third, Catmur, Mars, Rushworth \& Heyes (in prep) have provided evidence using paired-pulse TMS that, when mirror activation during action observation is mediated by the ventral premotor cortex, the countermirror effects of incompatible sensorimotor training on this activation are also mediated by the ventral premotor cortex. Therefore, it is likely that, in the present study, sensorimotor training acted directly on the human mirror system, rather than setting up parallel stimulus-response associations in dorsal premotor regions. 
Our findings support the view that the development of the mirror system depends on the same mechanisms of associative learning that mediate conditioning. If this is correct, associative learning theory may be applied to explain, predict and potentially to intervene in mirror system development. For example, the sensitivity of associative learning to contingency as well as contiguity can explain why most reported mirror neurons map an executed action (e.g. grasping) onto one or a small number of similar, observed actions (grasping only, or grasping plus holding). A monkey might, by chance, observe another individual performing a range of different actions while he is executing grasping movements. If contiguity was sufficient for mirror neuron development, all of these observed actions could acquire the potential to activate grasping motor neurons. However, if contingency is also required for mirror neuron development, only those observed actions that reliably predict, or are predicted by, action execution will acquire this potential. A predictive relationship of this kind - between similar observed and executed actions - is found in a limited range of circumstances; for example, when the learner observes their own body movements or, in the human case, is imitated by another agent (Ray \& Heyes, submitted).

Turning from explanation to prediction, the associative account of mirror neuron development predicts that 'counter-mirror neurons' or, more broadly, counter-mirror activation will be found in the ventral premotor cortex of participants who have not received special, laboratory-based training. This prediction follows from the evidence, reported here and elsewhere, that incompatible sensorimotor training in the laboratory can produce counter-mirror activation, combined with the observation that, in certain contexts, humans receive systematic incompatible sensorimotor experience in everyday life. For example, during coordinated instrumental action, the sight of another agent releasing an object reliably predicts the performance of a grasping action (Newman-Norlund, van Schie, van Zuijlen \& Bekkering, 2007; Van Schie, van Waterschoot \& Bekkering, 2008). Similarly, in social control situations, involving dominance and submission, expansive gestures by one interactant predict contraction movements by the other, and vice versa (Tiedens \& Fragale, 2003). If, as the current study suggests, the development of the mirror system depends on associative learning, experience of this kind should give rise to counter-mirror 
activation, in which the observation of one action systematically activates a motor representation of a complementary action. 


\section{REFERENCES:}

Bertenthal, B. I., Longo, M. R., \& Kosobud, A. (2006). Imitative response tendencies following observation of intransitive actions. Journal of Experimental Psychology: Human Perception and Performance, 32, 210-225.

Brass, M., Bekkering, H., \& Prinz, W. (2001). Movement observation affects movement execution in a simple response task. Acta Psychologica, 106, 3-22.

Calvo-Merino, B., Glaser, D. E., Grezes, J., Passingham, R. E., \& Haggard, P. (2005). Action observation and acquired motor skills: an fMRI study with expert dancers. Cerebral Cortex, 15, 12431249.

Calvo-Merino, B., Grezes, J., Glaser, D. E., Passingham, R. E., \& Haggard, P. (2006). Seeing or doing? Influence of visual and motor familiarity in action observation. Current Biology, 16, 1905-1910.

Catmur, C., Gillmeister, H., Bird, G., Liepelt, R., Brass, M., Brass, M., \& Heyes, C. (2008). Through the looking glass: counter-mirror activation following incompatible sensorimotor learning. Eurpoean Journal of Neuroscience, 28, 1208-1215.

Catmur, C., \& Heyes, C. M. (under review). Time course analyses confirm independence of automatic imitation and spatial compatibility effects.

Catmur, C., Walsh, V., \& Heyes, C. M. (2007). Sensorimotor learning configures the human mirror system. Current Biology, 17, 1527-1531. 
Catmur, C., Walsh, V., \& Heyes, C. M. (2009). Associative sequence learning: the role of experience in the development of imitation and the mirror system. Philosophical Transactions of the Royal Society Series B - Biological Sciences, 364, 2369-2380.

Catmur, C., Mars, R. B., Rushworth, M., \& Heyes, C. M. (under review). Making mirrors: premotor cortex stimulation enhances mirror and counter-mirror motor facilitation effects.

di Pellegrino, G., Fadiga, L., Fogassi, L., Gallese, V., \& Rizzolatti, G. (1992). Understanding motor events: a neurophysiological study. Experimental Brain Research, 91, 176-180.

Dickinson, A., \& Charnock, D. J. (1985). Contingency effects with maintained instrumental reinforcement. Quarterly Journal of Experimental Psychology, 37B, 197-416.

Dickinson, A., Watt, A., \& Griffiths, W. J. H. (1992). Free-operant acquisition with delayed reinforcement. Quarterly Journal of Experimental Psychology, 45B(3), 241-258.

Dimberg, U., Thunberg, M., \& Grunedal, S. (2002). Facial reactions to emotional stimuli: automatically controlled emotional responses. Cognition and Emotion, 16, 449-471.

Elsner, B., \& Hommel, B. (2004). Contiguity and contingency in action effect learning. Psychological Research, 68, 138-154.

Fadiga, L., Fogassi, L., Pavesi, G., \& Rizzolatti, G. (1995). Motor facilitation during action observation: a magnetic stimulation study. Journal of Neurophysiology, 73, 2608-2611.

Gallese, V. (2001). The "Shared Manifold" Hypothesis: from mirror neurons to empathy. Journal of Consciousness Studies, 8, 33-50. 
Gallese, V., Fadiga, L., Fogassi, L., \& Rizzolatti, G. (1996). Action recognition in the premotor cortex. Brain, 119, 593-609.

Gallese, V., \& Goldman, A. (1998). Mirror neurons and the simulation theory of mind-reading. Trends in Cognitive Sciences, 2, 493-501.

Gillmeister, H., Catmur, C., Liepelt, R., Brass, M., \& Heyes, C. M. (2008). Experience-based priming of body parts: A study of imitation and the mirror system. Brain Research, 1217, 157-170.

Grafton, S. T., Fagg, A. H., \& Arbib, M. A. (1998). Dorsal Premotor Cortex and Conditional Movement Selection: A PET Functional Mapping Study. Journal of Neurophysiology, 79, 1092-1097.

Grèzes, J., Armony, J. L., Rowe, J., \& Passingham, R. E. (2003). Activations related to mirror and canonical neurones in the human brain: an fMRI study. NeuroImage, 18, 928-937.

Halsband, U., \& Passingham, R. E. (1985). Premotor cortex and the conditions for movement in monkeys (Macaca fascicularis). Behavioural Brain Research, 18, 269-277.

Haslinger, B., Erhard, P., Altenmüller, E., Schroeder, U., Boecker, H., \& Ceballos-Baumann, A. O. (2005). Journal of Cognitive Neuroscience, 17, 282-293.

Hebb, D. (1949). The Organization of Behaviour. New York: Wiley.

Heyes, C. M. (2001). Causes and consequences of imitation. Trends in Cognitive Sciences, 5, 253-261. 
Heyes, C. M. (in press). Where do mirror neurons come from? Neuroscience and Biobehavioral Reviews.

Heyes, C., Bird, G., Johnson, H., \& Haggard, P. (2005). Experience modulates automatic imitation. Cognitive Brain Research, 22, 233-240.

Heyes, C. M., \& Ray, E. (2000). What is the significance of imitation in animals? Advances in the Study of Behaviour, 29, 215-245.

Iacoboni, M., Woods, R. P., Brass, M., Bekkering, H., Mazziotta, J.C., \& Rizzolatti, G. (1999). Cortical mechanisms of human imitation. Science, 286, 2526-2528.

Keysers, C., \& Gazzola, V. (2006). Towards a unifying neural theory of social cognition. Progress in Brain Research, 156, 379-401.

Keysers, C., \& Perrett, D. I. (2004). Demystifying social cognition: a Hebbian perspective. Trends in Cognitive Sciences, 8, 501-507.

Kornblum, S., Hasbroucq, T., \& Osman, A. (1990). Dimensional overlap: cognitive basis for stimulusresponse compatibility - a model and taxonomy. Psychological Review, 97, 253-270.

Leighton, J., \& Heyes, C. M. (in press). Hand to mouth: Automatic imitation across effector systems. Journal of Experimental Psychology: Human Perception and Performance

Mackintosh, N. J. (1975). A theory of attention: variations in the associability of stimuli with reinforcement. Psychological Review, 87, 532-552. 
Mitz, A. R., Godschalk, M., \& Wise, S. P. (1991). Learning-dependent neuronal activity in the premotor cortex: activity during the acquisition of conditional motor associations. Journal of Neuroscience, 11, $1855-1872$.

Newman-Norlund, R. D., van Schie, H. T., van Zuijlen, A., \& Bekkering, H. (2007). The mirror neuron system is more active during complementary compared with imitative action. Nature Neuroscience, 10, 817-818.

Oram, M., \&, Perrett, D. (1994). Responses of anterior superior temporal polysensory (STPa) neurons to "biological motion" stimuli. Journal of Cognitive Neuroscience, 6, 99-116.

Pearce, J. M., \& Hall, G. (1980). A model for Pavlovian learning: variations in the effectiveness of conditioned but not of unconditioned stimuli. Psychological Review, 87, 532-552.

Petrides, M. (1986). The effect of periarcuate lesions in the monkey on the performance of symmetrically and asymmetrically reinforced visual and auditory go, no-go tasks. Journal of Neuroscience, 6, 20542063.

Pinker, S. (1997). How the mind works. London, UK: The Penguin Press.

Press, C., Bird, G., Walsh, E., \& Heyes, C. M. (2008). Automatic imitation of intransitive actions. Brain \& Cognition, 67, 44-50.

Press, C., Gillmeister, H., \& Heyes, C. M. (2007). Sensorimotor experience enhances automatic imitation of robotic actions. Proceedings of the Royal Society of London: B, 274, 2509-2514.

Ray, E. D., \& Heyes, C. M. (under review). Imitation in infancy: The wealth of the stimulus. 
Rescorla, R. (1968). Probability of shock in the presence and absence of CS in fear conditioning. Journal of Comparative and Physiological Psychology, 66, 1-5.

Rescorla, R., \& Wagner, A. R. (1972). A theory of Pavlovian conditioning: variations in the effectiveness of reinforcement and non-reinforcement. In A. H. Black \& W. F. Prokasy (Eds.), Classical Conditioning II: current research and theory (pp.64-99). New York: Appleton-Century-Crofts.

Rizzolatti, G., \& Craighero, L. (2004). The mirror-neuron system. Annual Review of Neuroscience, 27, 169-192.

Rizzolatti, G., Fadiga, L., Gallese, V., \& Fogassi, L. (1996). Premotor cortex and the recognition of motor actions. Cognitive Brain Research, 3, 131-141.

Shanks, D. R. (1989). Selectional processes in causality judgment. Memory and Cognition, 17, 27-34.

Shanks, D. R., \& Dickinson, A. (1991). Instrumental judgment and performance under variations in action-outcome contingency and contiguity. Memory and Cognition, 19, 353-360.

Strafella, A. P., \& Paus, T. (2000). Modulation of cortical excitability during action observation: a transcranial magnetic stimulation study. Neuroreport, 11, 2289-2292.

Tiedens, L. Z., \& Fragale, A. R. (2003). Power moves: Complementarity in submissive and dominant nonverbal behavior. Journal of Personality and Social Psychology, 84, 558-568. 
Van Schie, H. T., van Waterschoot, B. M., \& Bekkering, H. (2008). Understanding action beyond imitation: reversed compatibility effects of action observation in imitation and joint action. Journal of Experimental Psychology: Human Perception and Performance, 34, 1493-1500.

Vogt, S., Buccino, G., Wohlschlager, A. M., Canessa, N., Shah, N. J., Zilles, K., Eickhoff, S. B., Freund, H. J., Rizzolatti, G., \& Fink, G. R. (2007). Prefrontal involvement in imitation learning of hand actions: effects of practice and expertise. Neuroimage, 37, 1371-1383.

Wagner, A. R. (1981). SOP: A model of automatic memory processing in animal behavior. In N. E. Spear \& R. R. Miller (Eds.), Information processing in animals: Memory mechanisms (pp. 5-47). Hillsdale, NJ: Lawrence Erlbaum Associates. 


\section{FIGURE LEGENDS:}

Figure 1 Examples of the stimuli used during training. Male compound stimulus presented on response-only trials (left); male compound stimuli presented on paired trials (central and right).

Figure 2 (a) Display sequence for paired trials employed in both the contingent and noncontingent training variants, for response mappings $1=$ make close hand response; $2=$ make open hand response. (b) Display sequence for an unpaired 'response-only' trial employed in the non-contingent training condition to degrade stimulus-response contingency.

Figure 3 Display sequences for test trials presenting (a) close-hand and (b) open-hand test stimuli. In one block of test trials participants made open-hand responses to stimulus onset; in the other they made close-hand responses.

Figure 4 Mean RTs observed in Experiment 1, plotted across the six training blocks where D1-2: day1 to day-2; and B1-B3: block-1 to block-3. Open circles represent the performance of the contingent training group, and filled circles represent the non-contingent group. Error bars represent standard error of the mean.

Figure 5 (a) Mean RTs on compatible and incompatible trials during the test phase for the contingent and non-contingent training groups. (b) Residual automatic imitation effects for the contingent and non-contingent training groups calculated by subtracting mean RTs on incompatible trials from mean RTs on compatible trials. Error bars represent the standard error of the mean.

Figure 6 Mean compatibility effects (ms) plotted for each of the four quartiles of the RT distributions. Q1 denotes fastest quartile; Q2 the second fastest quartile; Q3 the third fastest; and Q4 the slowest quartile.

Figure 7 Examples of the stimuli used for the signaled training group. Male warning stimulus (left); male irrelevant stimulus with ' 1 ' imperative stimulus (central).

Figure 8 (a) Display sequence for a paired trial employed in the signaled training condition, where participants instructed $1=$ close hand; 2 = open hand. (b) Display sequence for an 
unpaired trial employed in the signaled training condition.

Figure 9 Mean RTs observed in Experiment 2, plotted across the six training blocks where D1D2: day-1 to day-2, and B1-B3: block-1 to block-3. Open circles represent the performance of the contingent training group; filled circles represent the non-contingent group; and triangles represent the signaled group. Error bars represent standard error of the mean.

Figure 10 (a) Mean RTs on compatible and incompatible trials during the test phase for the contingent, non-contingent and signaled training groups. (b) Mean automatic imitation effects for the contingent, non-contingent, and signaled training groups. Error bars represent the standard error of the mean.

Figure 11 Mean automatic imitation effects (ms) observed in Experiment 2 plotted for each of the four quartiles of the RT distributions. Q1 denotes fastest quartile; Q2 the second fastest quartile; Q3 the third fastest; and Q4 the slowest quartile. Error bars represent the standard error of the mean. 
FIGURES:

Figure 1:
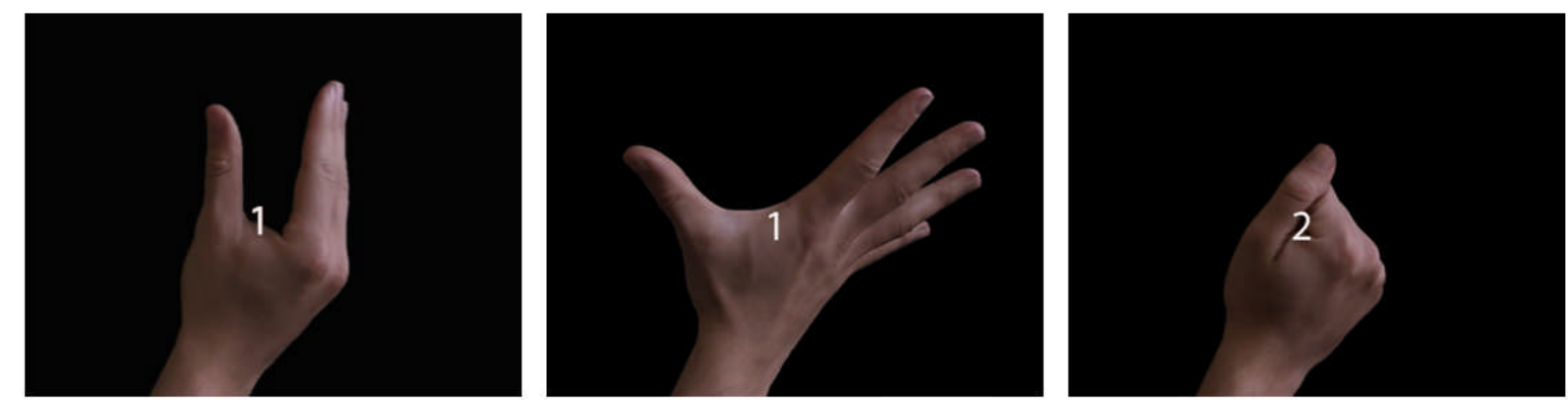

Figure 2:

a)
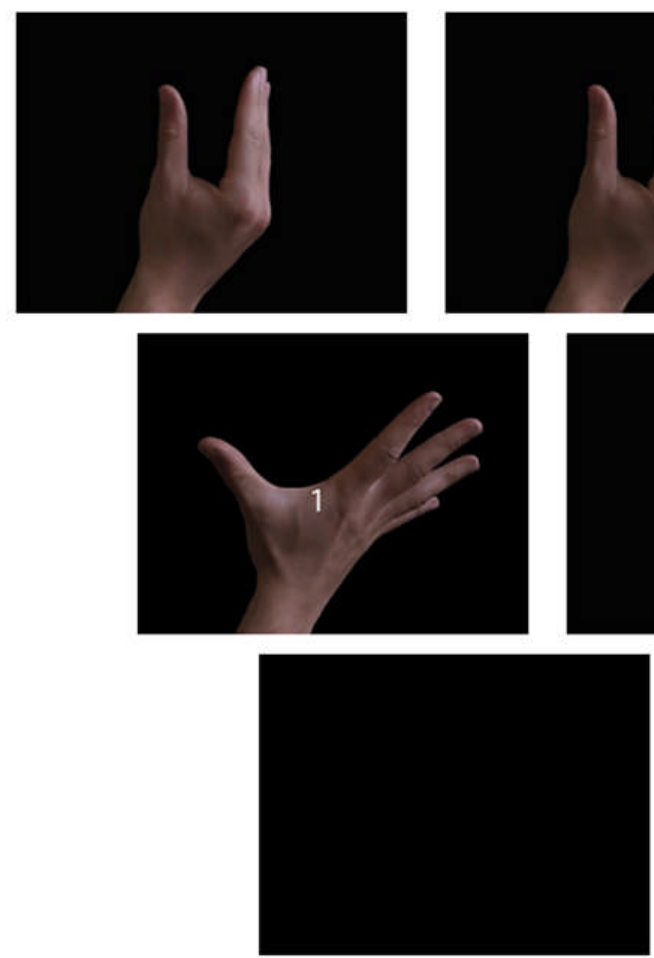

b)
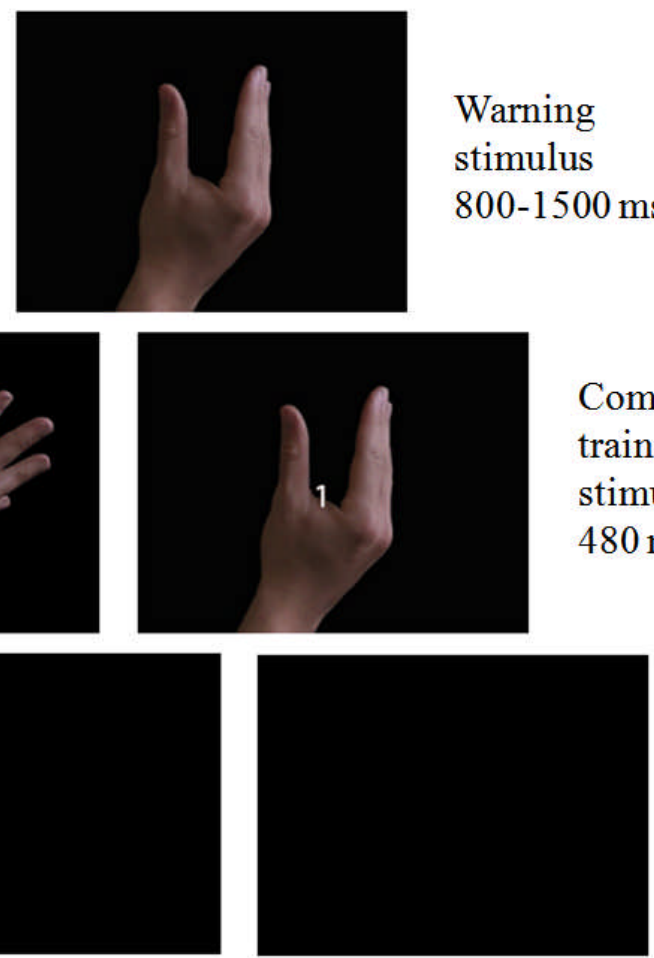

Warning

stimulus

800-1500 ms
Participant's

hand in starting position

\begin{tabular}{|c|c|}
\hline $\begin{array}{l}\text { Blank } \\
\text { screen } \\
3000 \mathrm{~ms}\end{array}$ & $\begin{array}{l}\text { Participant } \\
\text { returns hand } \\
\text { to starting } \\
\text { position }\end{array}$ \\
\hline
\end{tabular}


Figure 3:

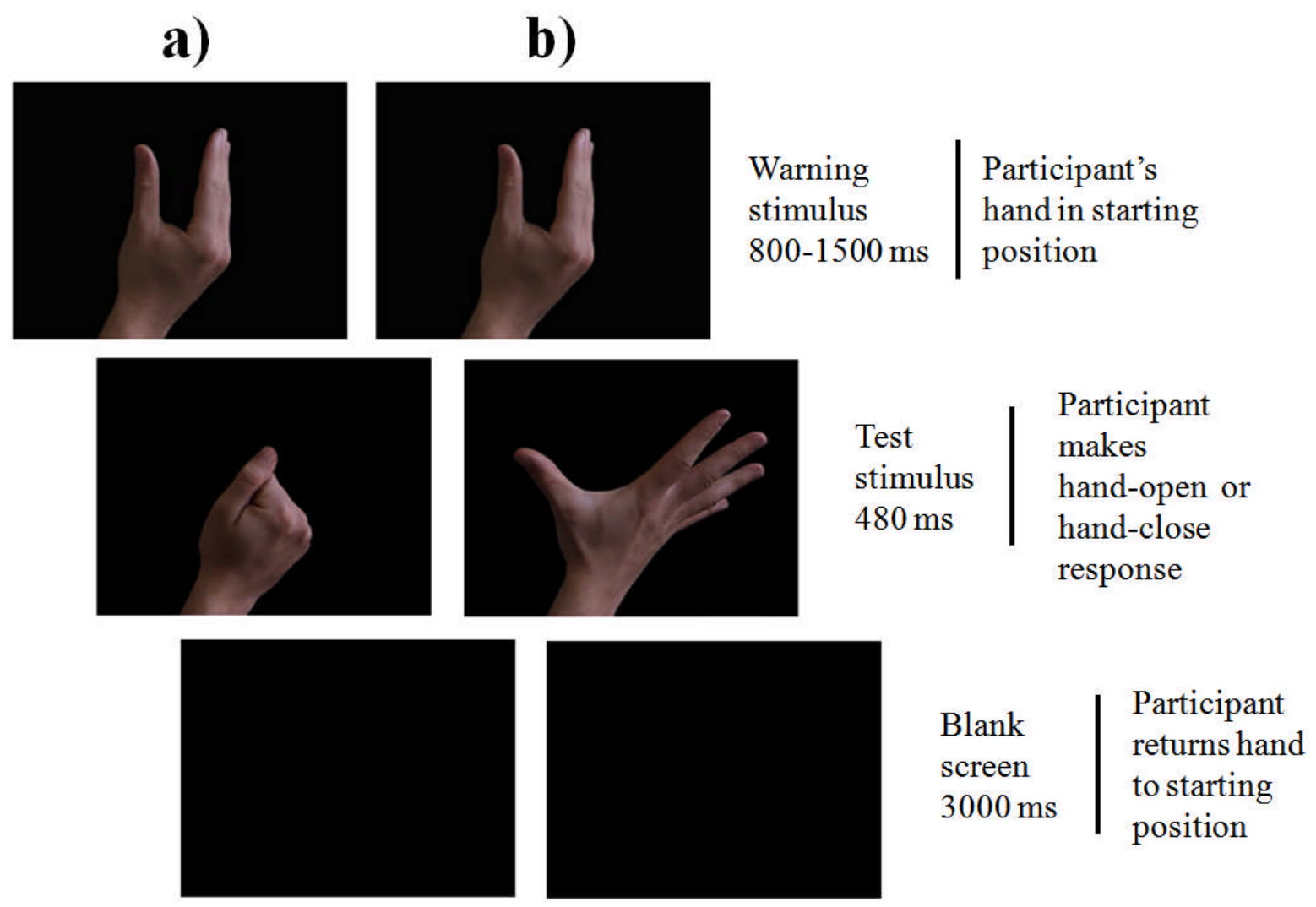


Figure 4:

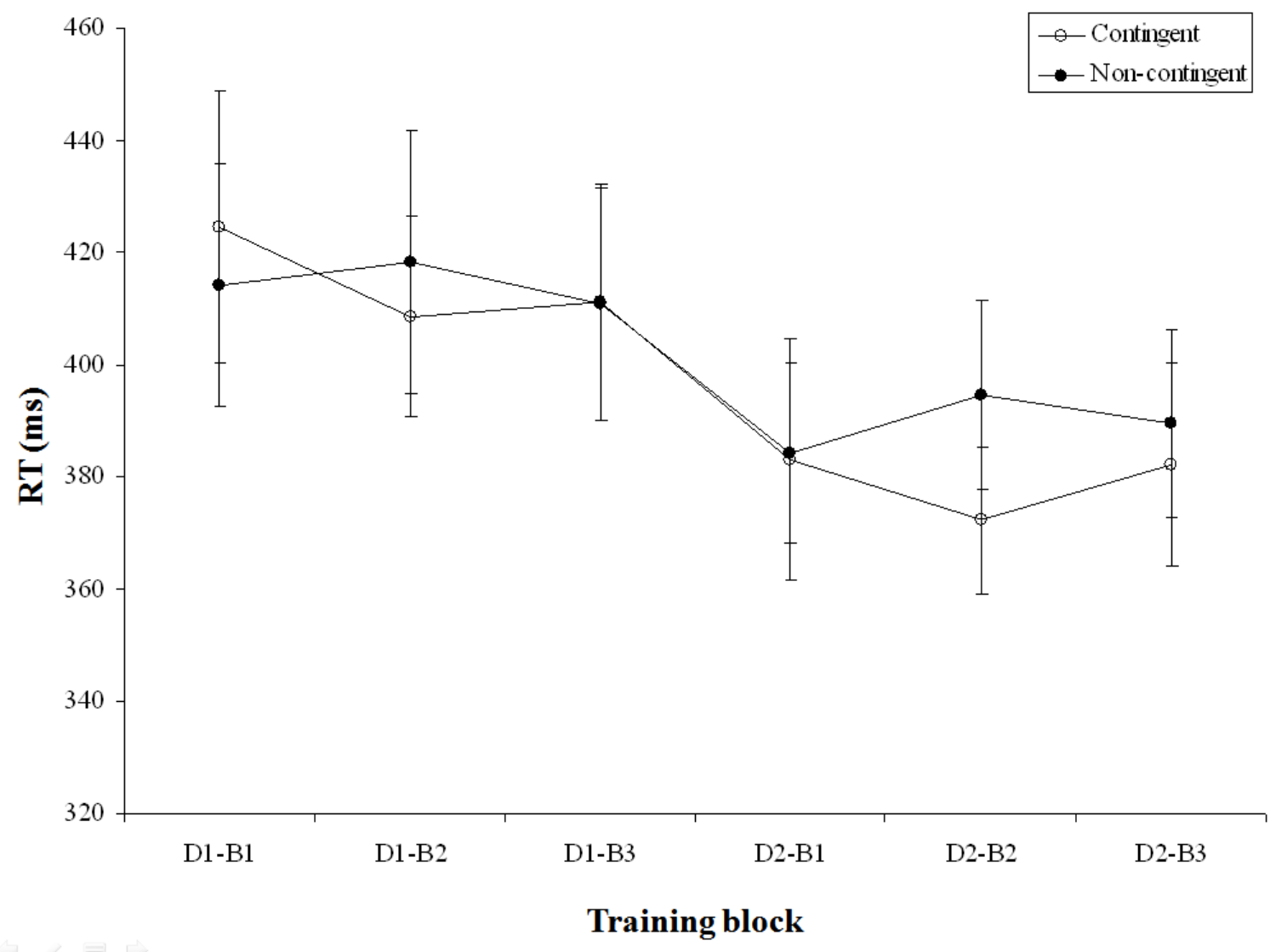


Figure 5:

(a)

$\square$ Compatible Trials

- Incompatible Trials

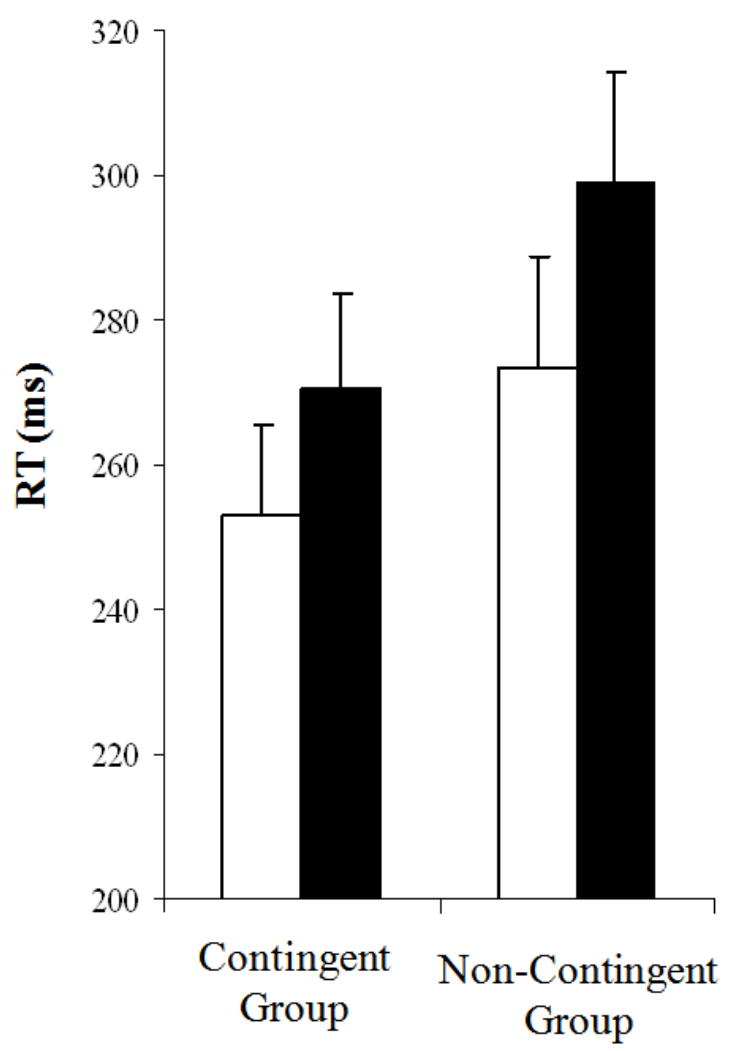

(b)

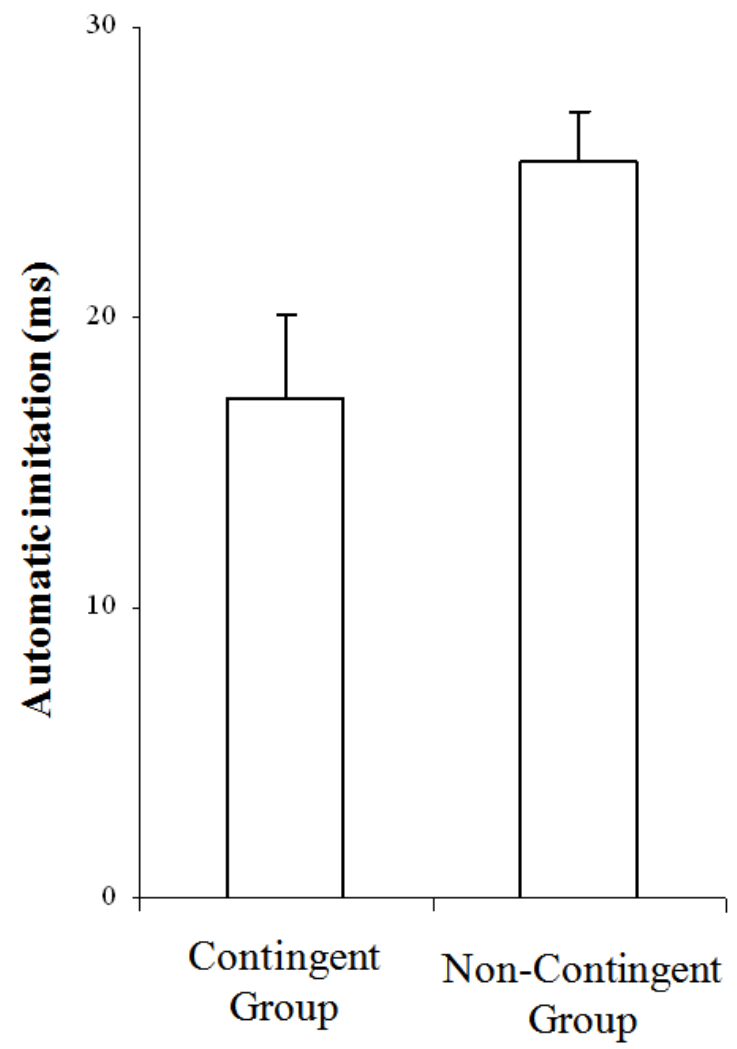


Figure 6:

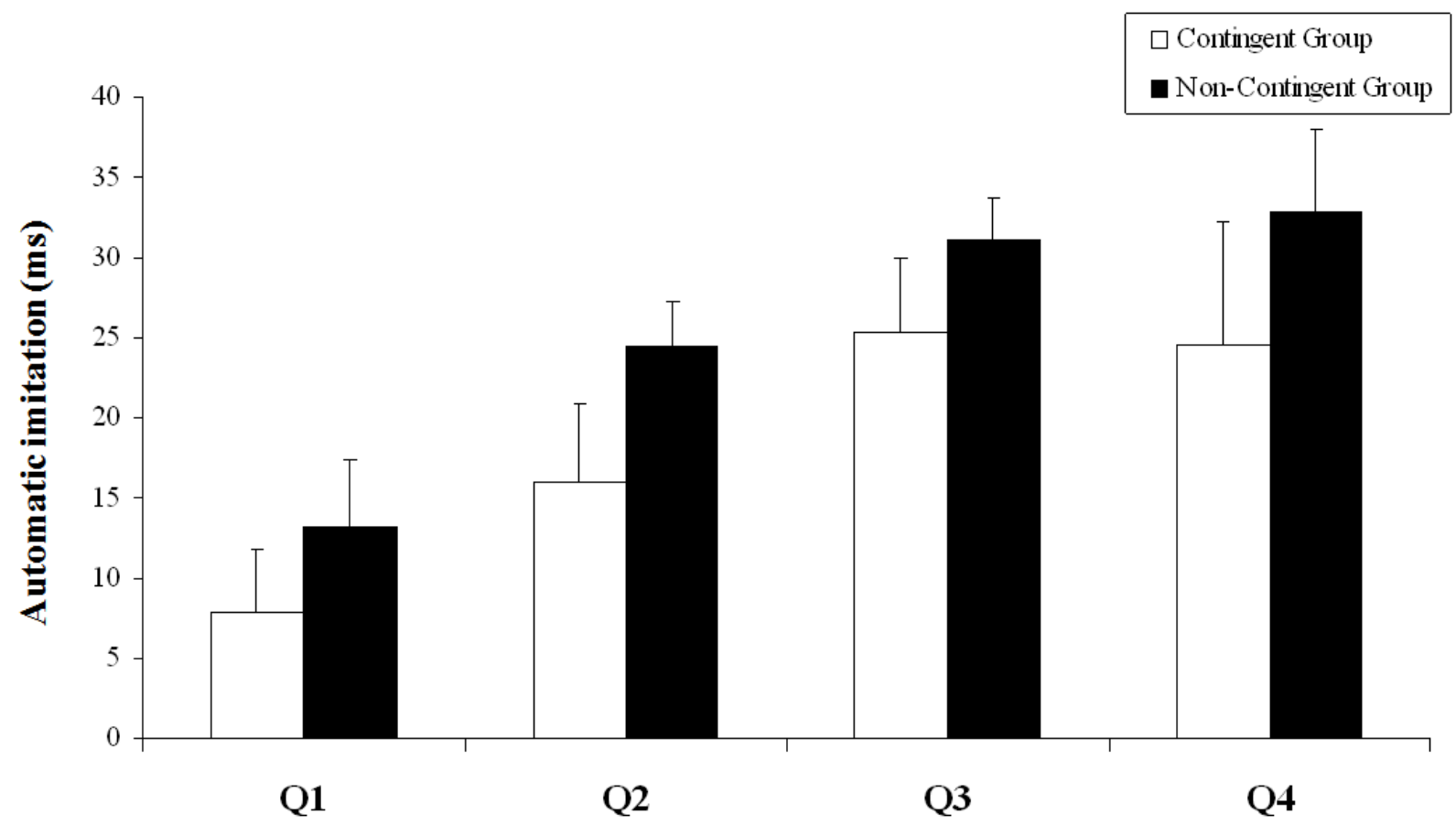

Figure 7:
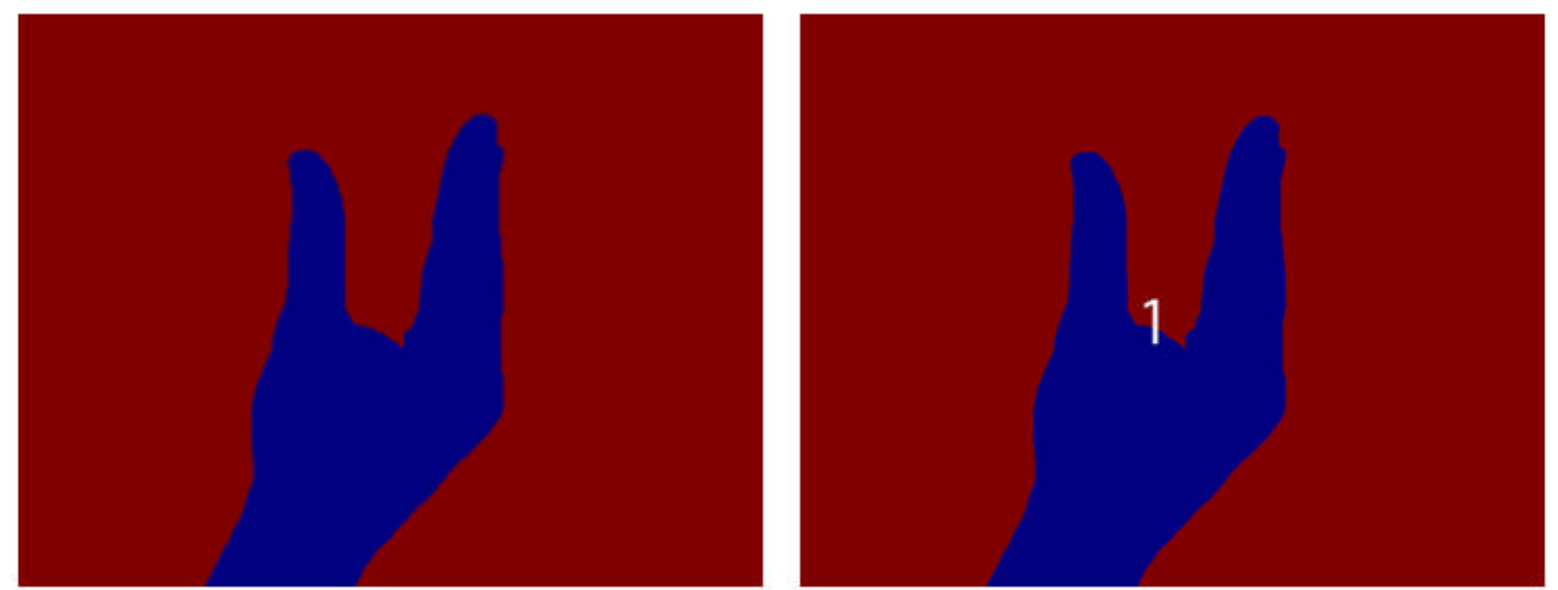
Figure 8:

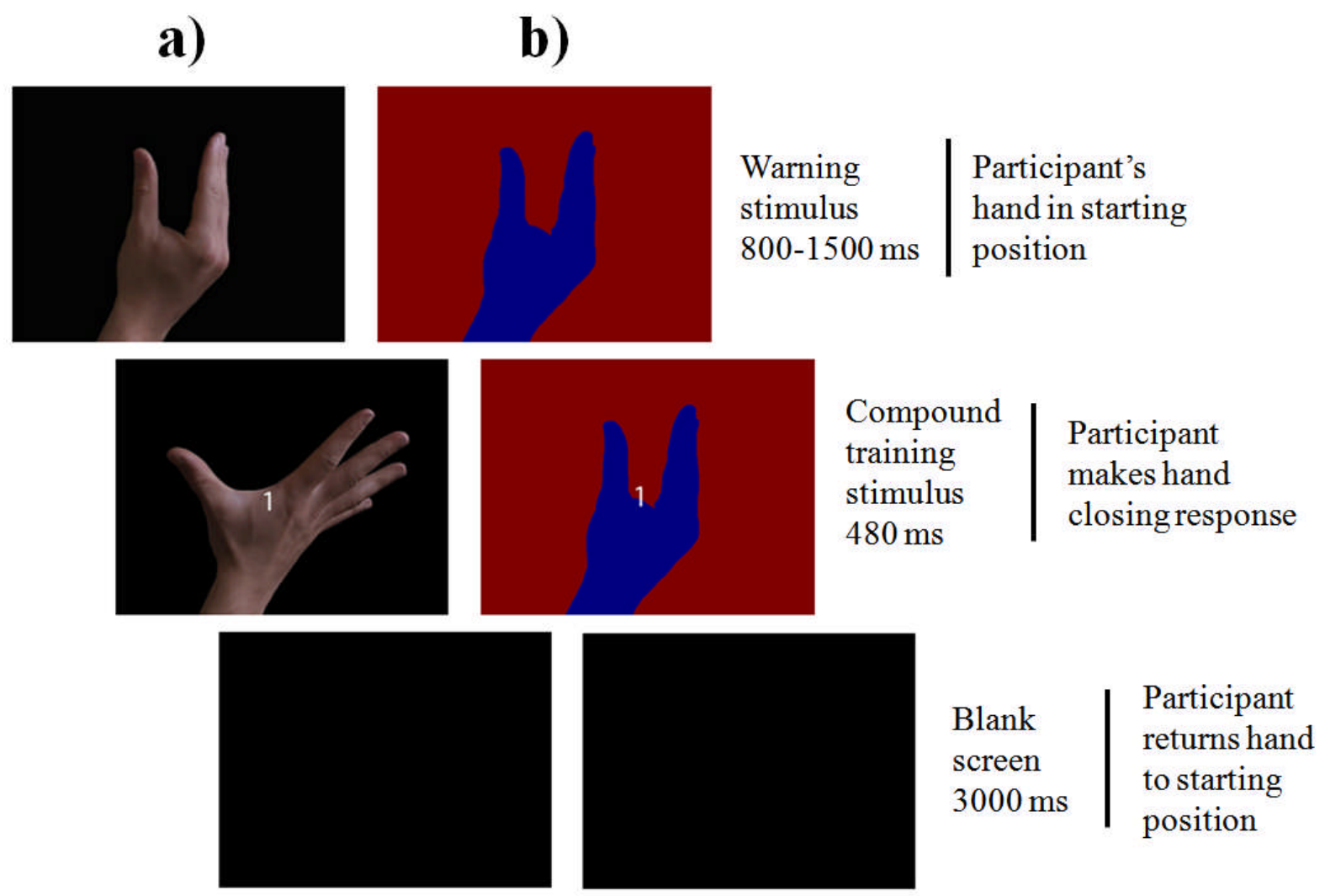


Figure 9:

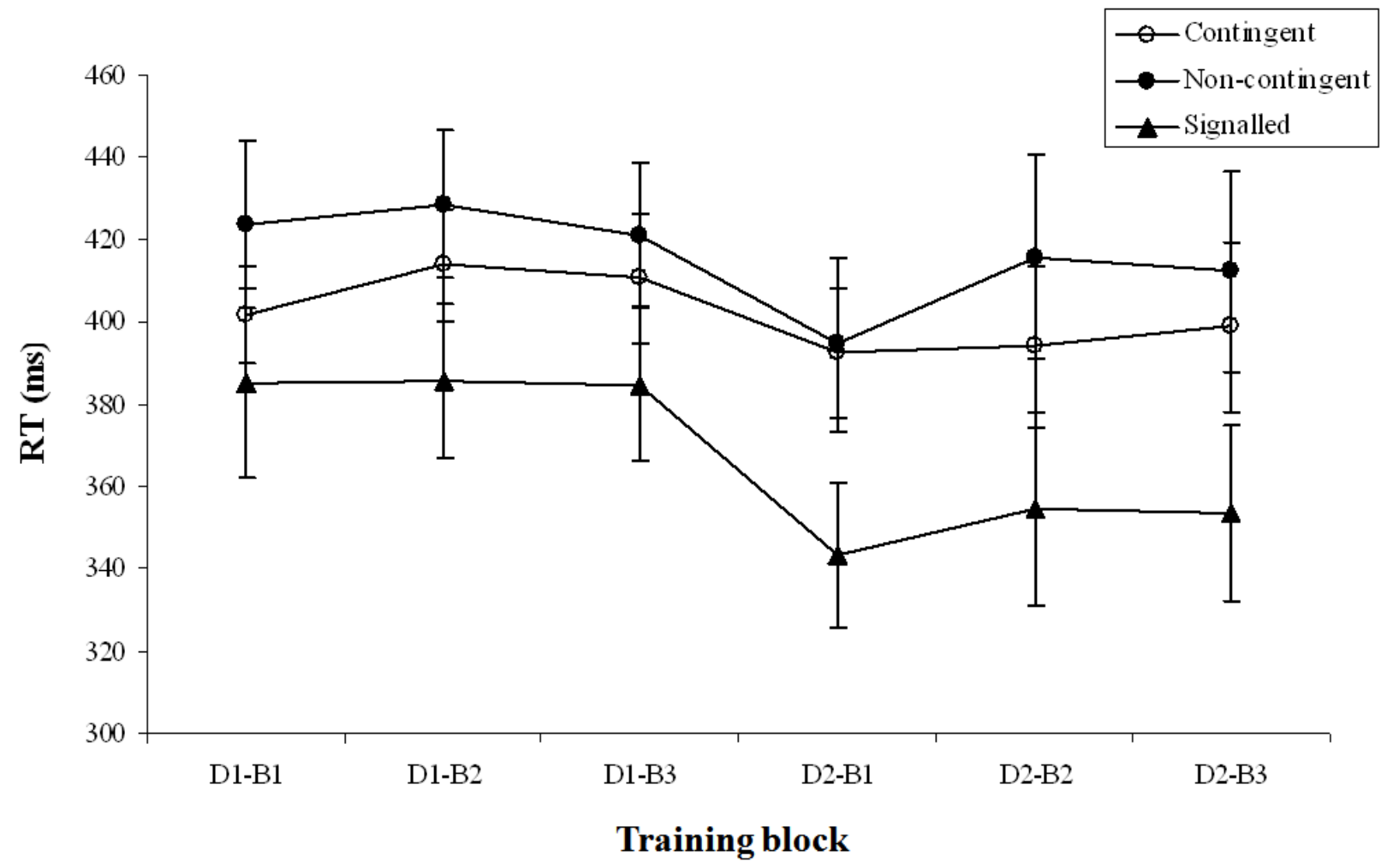


Figure 10:

(a)

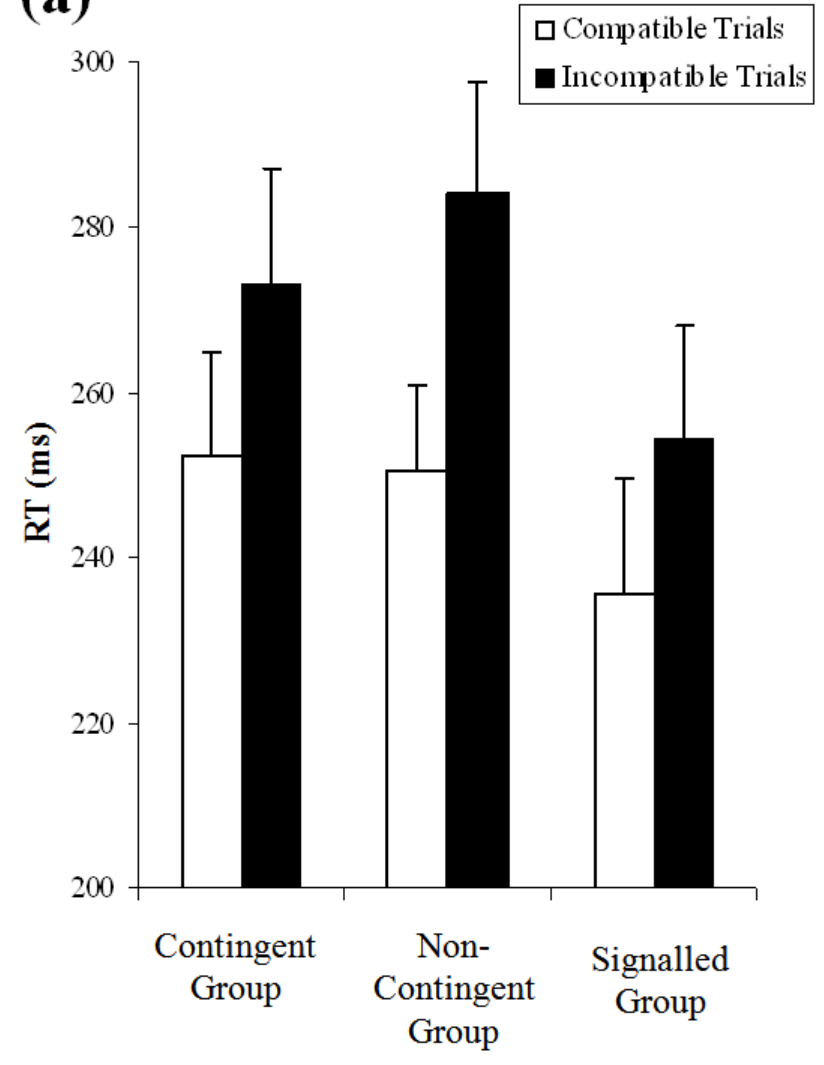

(b)

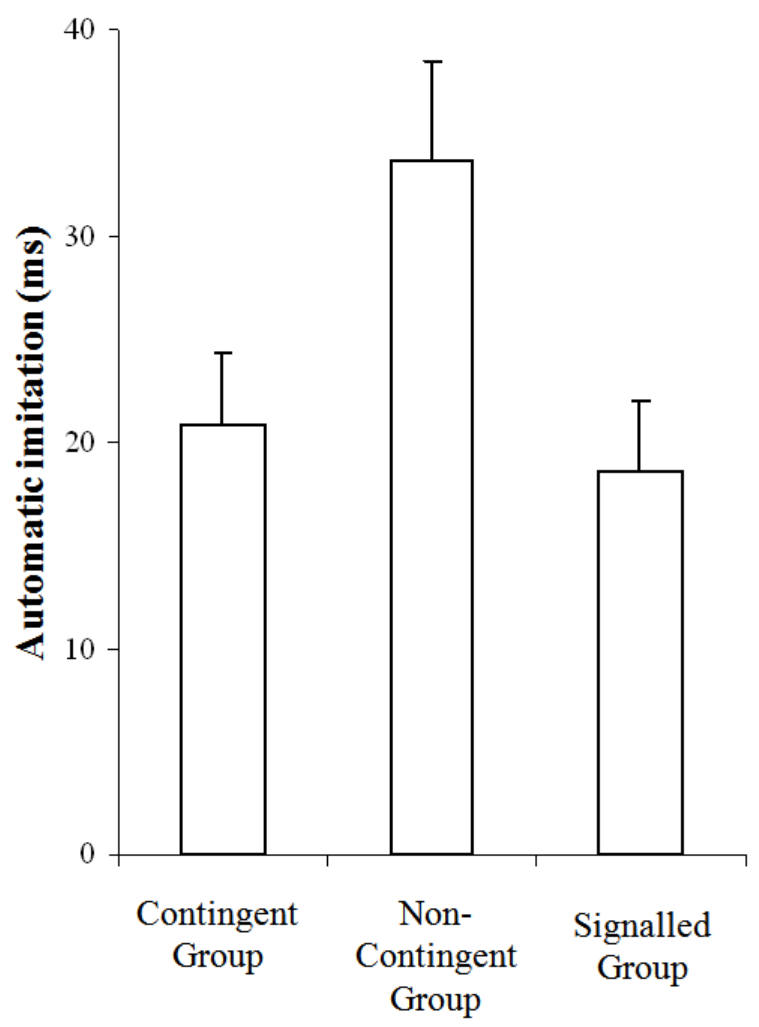


Figure 11:

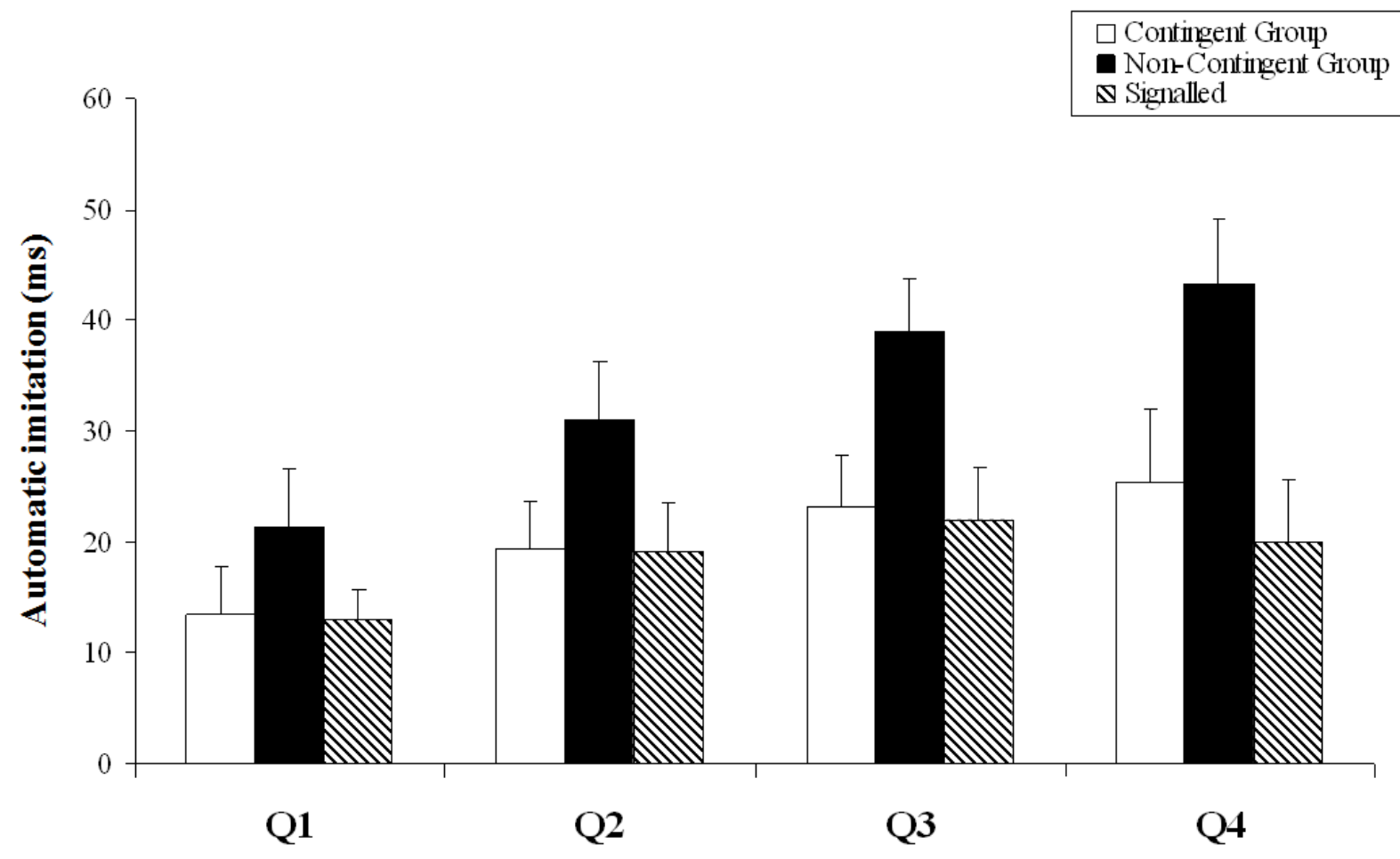

